\title{
Web application for display and analysis of clinical data from a home telemonitoring platform
}

\author{
A. Tura ${ }^{1}$, P. Santini ${ }^{2}$, D. Longo ${ }^{2} \&$ L. Quareni $^{2}$ \\ ${ }^{I} I S I B-C N R$, Padova, Italy \\ ${ }^{2}$ QBGROUP, Padova, Italy
}

\begin{abstract}
We designed and developed a home telemonitoring instrument, and related Web application for management of clinical data. The instrument directly measures important physiological parameters (blood oxygen saturation, pulse rate, and respiratory parameters related to the use of pulmonary ventilators). The instrument also connects to external instruments (spirometer, capnometer, noninvasive blood pressure measuring device), thus collecting the corresponding clinical parameters. The instrument has a flexible architecture, which allows insertion of the necessary boards only, depending on the parameters that need to be measured and the external instruments to be interfaced for each patient. The measured and collected data are transmitted to a Web server, through direct connection to a local Internet POP (thanks to the TCP/IP protocol implemented at the instrument firmware level), thus avoiding long distance calls possibly occurring in traditional modem-to-modem configurations. Transmitted data are made available for consultation through a Web application from the health professionals, wherever they are (if an Internet connection is available). The advantage of using a Web application instead of a traditional one is that all installation problems are avoided, and the latest application version is immediately available for all the users. Within the Web application, the clinical data can be displayed and analyzed both in numeric and graphic format. It is also possible to mark some portions of data with proper indicators: for instance, for saturation curves all the desaturation events can be marked manually or after identification through an automatic algorithm. Furthermore, the Web application allows possible definition of a new instrument setup, which can be transmitted to the instrument thanks to the bidirectional connection with the Web server. The system is under test on fifteen patients with amyotrophic lateral sclerosis and respiratory problems. In future, the system could also be used for telemonitoring of different categories of patients with chronic pathologies.
\end{abstract}

Keywords: Internet, telemedicine, telecare, e-health, chronic pathologies. 


\section{Introduction}

The significant development in telecommunication technologies in the last years has made the Internet an effective and convenient solution for telemedicine applications. A traditional field for telemedicine, which is having great benefit from easy and affordable access to the Internet, is that of teleconsultation or second opinion, which provides the opportunity for health care professionals to interact with colleagues in every part of the world [1-3]. A less established, but emerging telemedicine field, which is using the Internet increasingly, is that of telemonitoring of subjects located outside the health care structures. That includes both monitoring of subjects in an outdoor environment in even moving conditions (such as for sport [4] or military [5] applications) and, more frequently, monitoring of patients with chronic pathologies at their homes [6-10]. A specific category of disease, where home telemonitoring can be convenient, is that of chronic respiratory pathologies, such as asthma [6] or obstructive pulmonary disease [11]. In this study, we present an Internet-based telemonitoring system for management of patients with respiratory problems, based on a recently developed telemonitoring instrument.

\section{The home telemonitoring platform}

The core of the home platform is a telemonitoring instrument, which is able to measure some important physiological parameters. Furthermore, the instrument can be connected to other external instruments possibly present at the patient's home. Parameters directly measured by the instrument are: i) blood oxygen saturation; ii) pulse rate; iii) respiratory parameters from pulmonary ventilators. Parameters collected through connection to the external instruments are those provided by: iv) a spirometer; v) a capnometer; vi) a non-invasive blood pressure (NIBP) measuring device. When interfaced to the external instruments, our instrument acts as a sort of digital recorder.

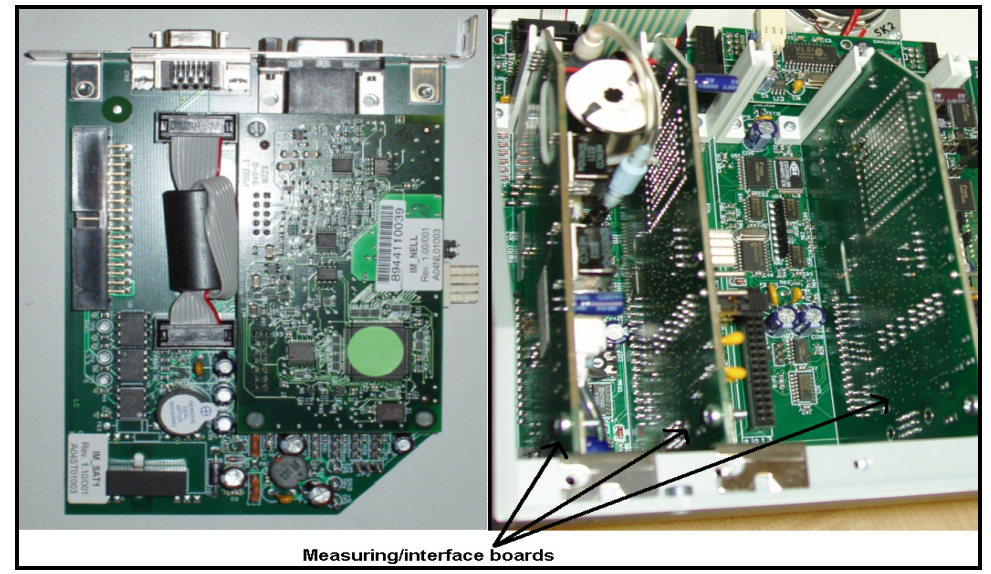

Figure 1: One measuring board (left), and boards inserted in the slots (right). 
The instrument is made of a main board, with five slots on it. These slots allow insertion of five different electronic boards for parameter measurements or for interface to the external instruments. More specifically, there are two measuring boards and three interface boards. The former are the saturation board, for measurements of parameters i)-ii), and the ventilation board, for parameters iii); the latter are the spirometer, capnometer, NIBP boards, for interfacing to external instruments iv), v), vi), respectively. An example of board is depicted in Figure 1, which also shows the internal part of the instrument with the slot architecture.

This slot-based architecture allows great flexibility: only the measuring/interface boards necessary for the specific patient are mounted, depending on the parameters to be measured and external instruments that he is using. It is worth noting that the ventilation board allows connection to all the ventilators (also the old ones without any electrical communication port), since the interface is realized through an air flow sensor inserted into the patient's circuit, i.e. inside the tube that brings air from the ventilator to the patient. Conversely, each interface board allows connection to a specific commercial external instrument, although future connection to other instruments could only require intervention at firmware and not hardware level. Similarly, the saturation board at the moment requires a saturation probe from a specific manufacturer, but it is possible to choose among several probes (finger, ear, forehead probes).

The instrument is equipped with an internal modem, which allows direct connection to the Internet through TCP/IP protocol, implemented at firmware level. Therefore, the clinical data measured or collected by the instrument can be downloaded to a Web server, and hence made available for consultation to the health professionals through the Internet. Thanks to a full-duplex Ethernet interface the Web server could also be reached through a LAN (Figure 2).
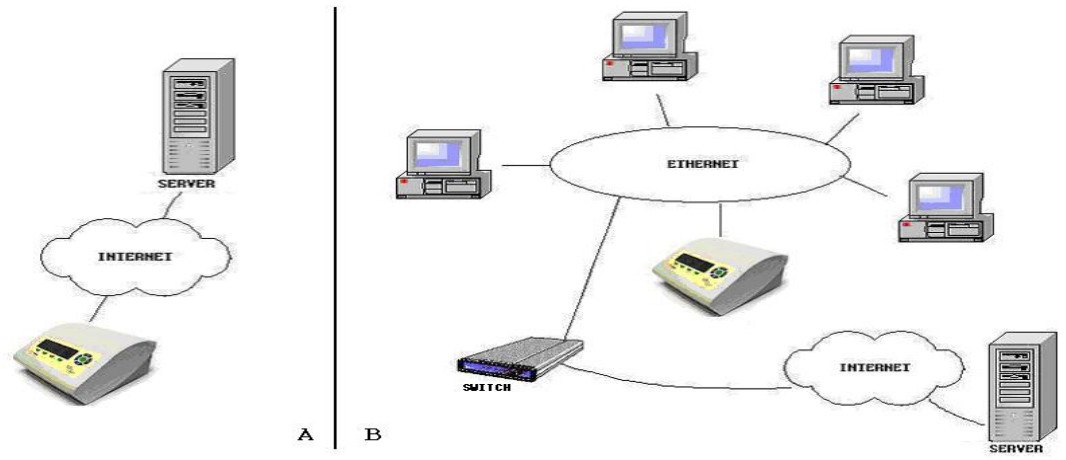

Figure 2: Connection of the instrument to the Web server: through internal modem (A), or through LAN (B).

It must be noted that, differently from many telemedicine instruments that transmit data remotely through modem-to-modem connection, our instrument directly connects to a local Internet POP (due to the internally implemented 
TCP/IP protocol), thus avoiding some problems such as possible occurrence of long distance calls.

\section{Communication between telemonitoring instrument and Web server}

Communication between the instrument and the Web server allows collection of clinical data from the patients, and consequent consultation through a Web application. Communication occurs through exchange of messages between the instrument (acting as client) and the server. Messages are made of a header, a message type field, a command field, a payload (with relative length at the beginning), and a CRC field to check data integrity. The first message that the instrument sends is of "Connect" type. There are three main reasons for asking connection to the server, specified by different command fields: i) automatic transmission: the instrument has to send some clinical data, according to a prescribed protocol (for instance, transmission of data at a given time, everyday); ii) manual transmission: the instrument has to send some clinical data upon request (through the instrument keyboard) of the patient or of one operator at the patient's home; iii) patient synchronisation: synchronisation of patient's data on the instruments with patient's data at the Web server database. The server acts as master of the communication: depending on the reason for connection, it sends to the instrument the proper messages and waits for responses. An example of message exchange is reported in Figure 3: in that case, the instrument sent its current setup and the clinical data stored in its memory, and received from the server a new setup, as well as a questionnaire to be completed by the patient.

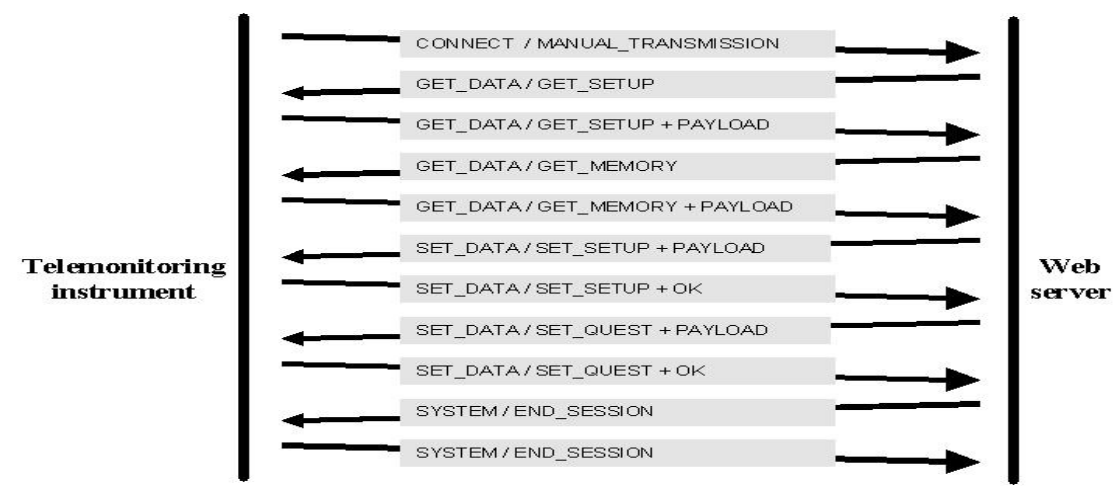

Figure 3: Message exchange during a communication session.

\section{Display and analysis of clinical data through the Web application}

The Web application associated with the home telemonitoring platform, running on the Web server, allows the display and analysis of the clinical data, both in 
numeric and graphic format. The advantage of using a Web application compared to a traditional application is relevant: i) access to the data from every personal computer or similar device (the only condition is to be equipped with Internet connection); ii) no problems related to the installation of the application on each single computer; iii) immediate availability of the latest version of the application. After authentication in the initial window of the Web application, the operator gets access to the main window, reported in Figure 4.

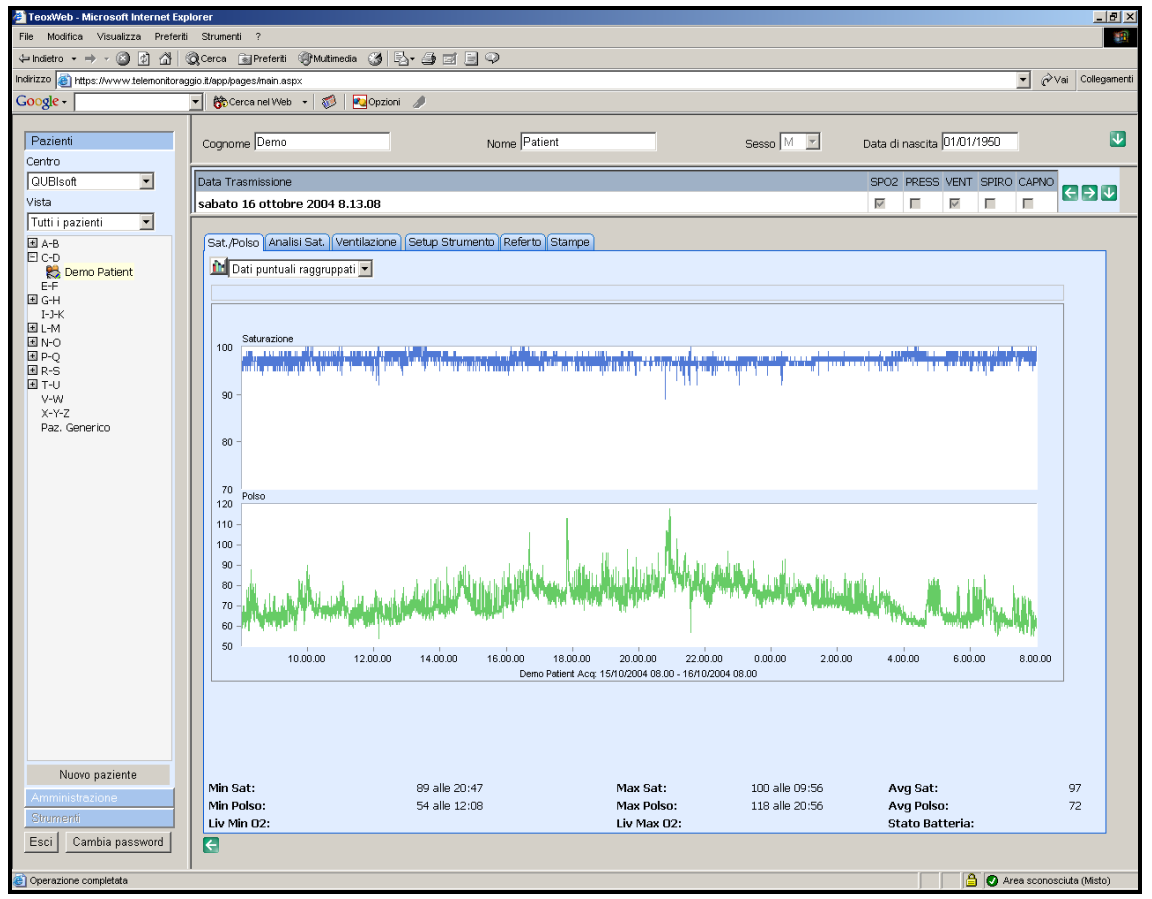

Figure 4: Main window of the Web application.

The window is divided in four areas:

1) Function Selection Area (left): the operator can select the function to be performed: selection of one patient, management of telemonitoring instruments, administration functions (for instance, creation of new operators)

2) Patient Management Area (right, top): the operator can insert, modify, or display the personal data of the selected patient

3) Transmission List Area (right, middle): the list of all the transmissions by the telemonitoring instrument is reported for the selected patient

4) Data Display Area (right, bottom): data of the selected transmission are displayed.

The described areas can be expanded or reduced through arrows. In Figure 4 data from the instrument saturation board, i.e. blood oxygen saturation and pulse rate, 
are reported. Examples of display of different types of clinical data are reported in Figure 5 and Figure 6.

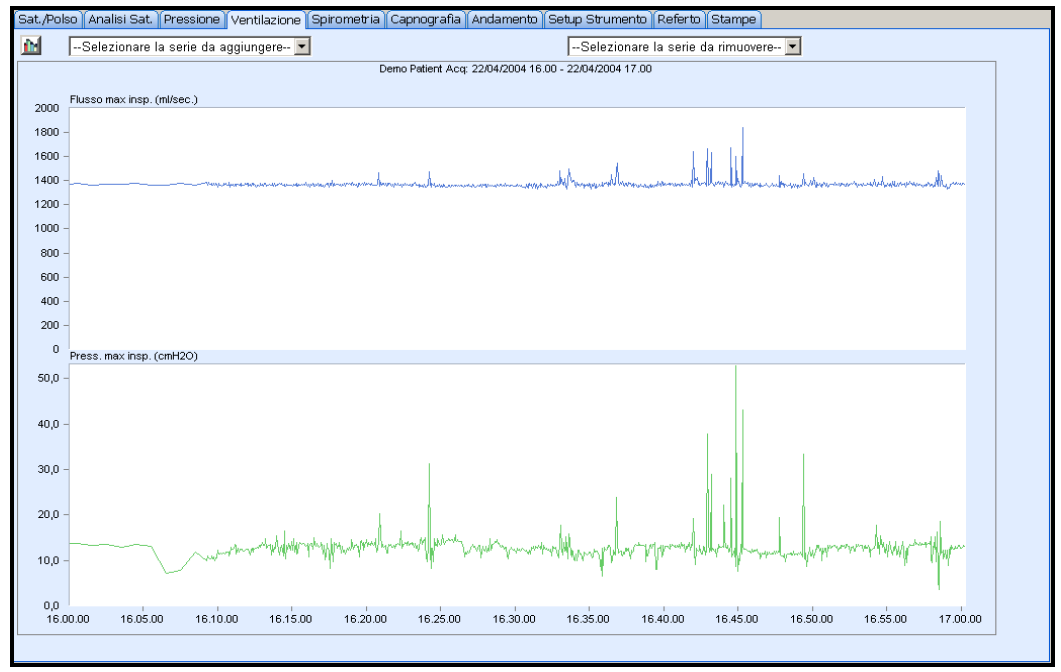

Figure 5: Display of data from the ventilation board: maximum inspiration flow and pressure.

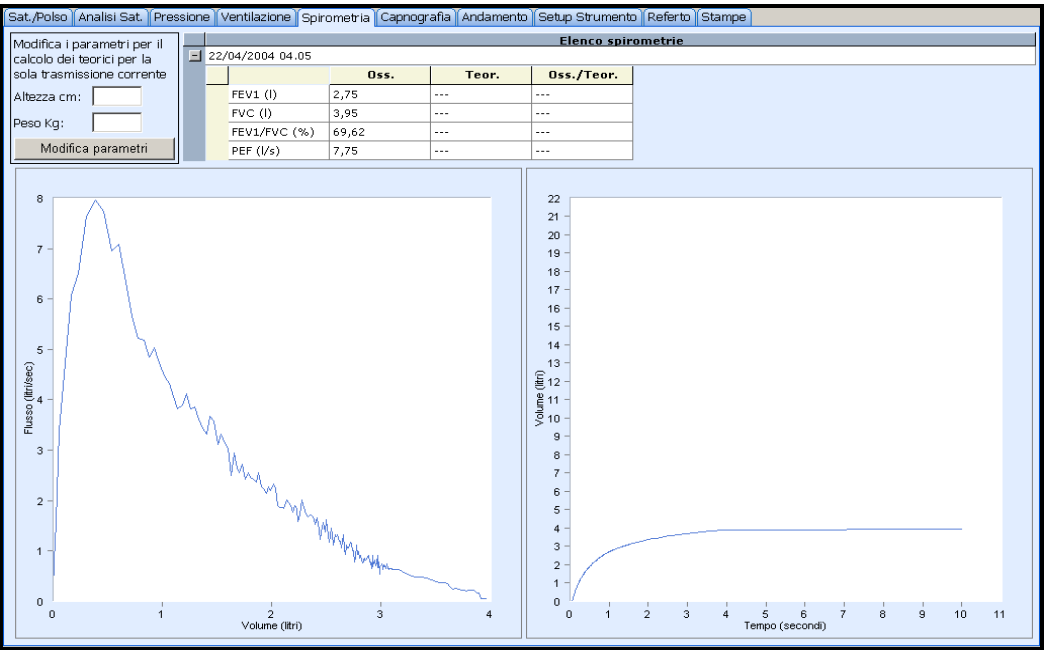

Figure 6: Display of data from the spirometer board: flow and volume during the test, and table with most common parameters in spirometry. 
It must be noted that the operator can enter an advanced data display mode, thus allowing zoom operations and other strategies for deep analysis of the data. For instance, in the case of the saturation and pulse data, it is possible to mark some portion of the graphics as artefacts, or as desaturation events. An algorithm for automatic detection of desaturation events is also available. A report window is present where the operator can write his comments on the current patient's health condition, and can set a level of attention indicator based on colour codes.

\section{Telemonitoring instrument setup from the Web application}

The communication between the telemonitoring instrument and the Web server is bidirectional. Thus, from the Web application it is possible to send to the instrument some information, such as a new setup. In the setup several parameters are defined, especially related to the acquisition modality of the clinical data (for instance, time, interval length, and frequency of acquisition for the saturation and pulse data). In Figure 7 it is in fact reported the Web application window where the setup for saturation can be defined. It also includes setup parameters for acquisition of the $\mathrm{O}_{2}$ level in an $\mathrm{O}_{2}$ unit possibly present.

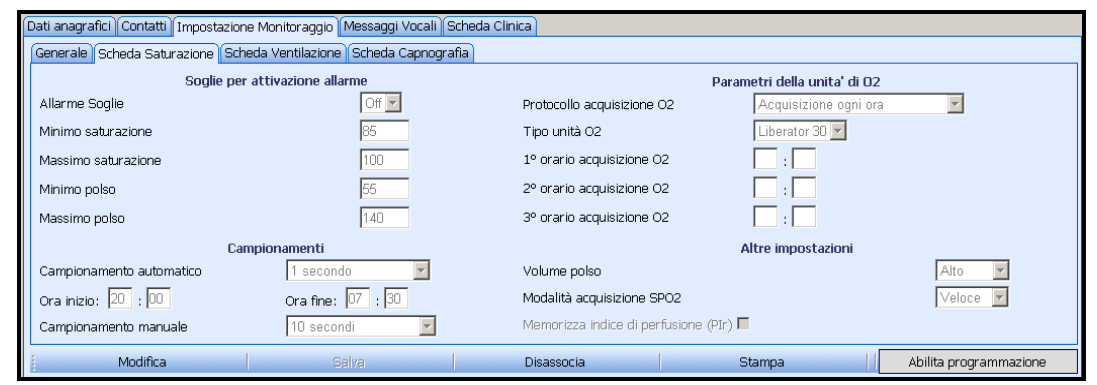

Figure 7: Window for setup of saturation board parameters.

\section{Security issues}

The whole project has been designed with high attention to the security issues, which are very important when telemedicine applications use the Internet [12]. First, the communication between the telemonitoring instrument and the Web server is encrypted, through a high security algorithm (symmetric algorithm Rijndael, with 128 bit key). Furthermore, each operator can use his password for access to the Web application for no more than three months: afterwards, the application forces the operator to change the password. It must be noted that each operator will never be allowed to use one password already used in the past. Moreover, if one operator does not access the application for more than six months, it is automatically disabled: to get access again, the operator will have to ask the system administrator for a new password. Finally, great attention has 
been given in the definition of different authorisation profiles. Each operator can in fact have one or more of the following rights: i) display; ii) create; iii) update; iv) delete. Obviously, if one operator has one of the rights ii)-iv), he automatically will have right i). Furthermore, these different rights can be selectively applied to the different functions that can be performed within the Web application, i.e. management of: i) patients' personal data; ii) patients' clinical data; iii) telemonitoring instruments; iv) administrative issues. It is also possible to provide rights only on some specific groups of patients. A Web application window where these rights can be defined is reported in Figure 8.

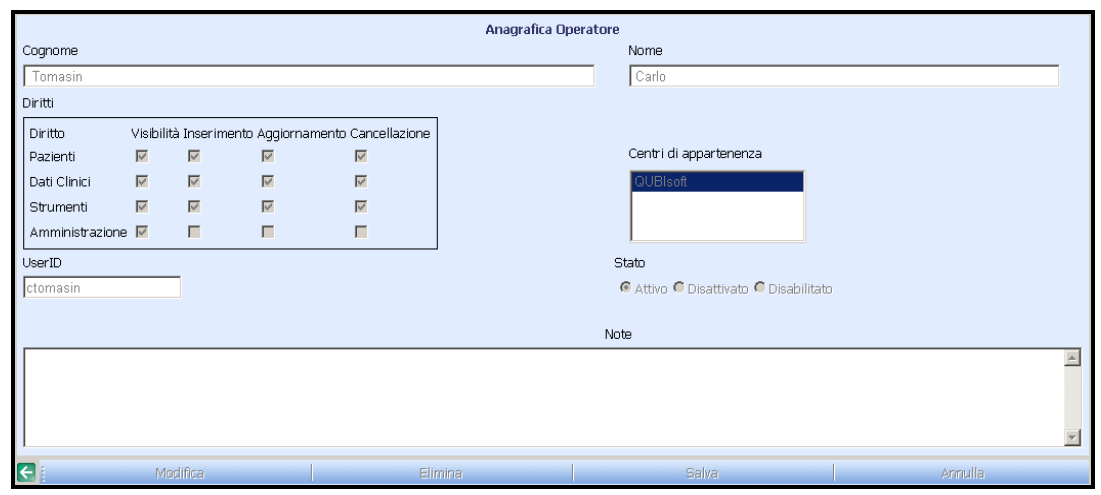

Figure 8: Definition of operator's right within the Web application.

\section{Discussion and conclusions}

Telemedicine applications are rapidly increasing due to the fast development of electronic and telematic networks and systems. Home telemonitoring is a promising field within telemedicine: although its diffusion is still limited by problems of reimbursement, some health care systems are changing their policies on that [13]. One application of home telemonitoring is that of chronic respiratory diseases. We have in fact realised an Internet-based platform for monitoring of this type of patients. A telemonitoring instrument was designed and developed, which is able to measure important physiological parameters related to respiratory problems. Furthermore, the instrument is able to connect to external instruments, possibly present at the patient's home, to collect other parameters. The telemonitoring instrument is modular, since it is possible to mount only the boards necessary for the specific clinical needs of each patient (in a PC-like fashion), thus allowing significant cost saving. Another interesting feature of the instrument is the ability to directly transmit all the measured or collected data, without the need of other devices (such as computers or similar devices), which, conversely, are used in many home telemonitoring applications [6-10]. In other telemonitoring applications a direct connection of one instrument with a remote data center was realised, but the communication was usually modem-to-modem. That requires a sufficiently large stack of modems at the 
remote data center, to fulfil possible contemporary calls of instruments from the patients' homes. Conversely, our instrument directly connects to Internet through a local POP, and hence no stack of modems is required remotely, but only an Internet connection with sufficient bandwidth. Moreover, this solution leads to local calls only, whereas the modem-to-modem solution could lead to long distance calls, depending on the patient's home geographical position with respect to the remote data center position.

In our platform, once the patients' data have been transmitted from the instrument to the remote data center, they can be accessed by the health professionals through a Web application, provided by a proper server. In this context, the advantage of a Web application is remarkable, compared to a traditional application installed on each computer: installation problems are in fact avoided, and the updated version of the application is immediately available for all the operators. It is worth noting that our Web application allows not only display and analysis of patients' clinical data, but also remote instrument management: for instance, it is possible to send a new setup for all the instrument parameters, such as those related to measure and/or collection of the clinical data. Furthermore, it could also be possible to send to the instrument a new firmware version: although this is not a common need, the instrument is technically able to receive new firmware, and to reprogram itself.

At the moment, the described telemonitoring system is under test. Fifteen patients with amyotrophic lateral sclerosis and with respiratory problems are participating to the preliminary trial. However, in the future the system could be extended to several categories of subjects with respiratory problems, such as patients with cystic fibrosis, or brain-injured children [14-15]. Furthermore, thanks to the flexible architecture of the telemonitoring instrument, the system could also be applied to other patients with chronic pathologies other than respiratory ones, such as patients with diabetes or cardiovascular problems: from the hardware point of view, that would in fact require the development of a new measuring or interface board, without affecting the rest of the instrument. In conclusion, we are confident that home telemonitoring solutions will improve the quality of life of several categories of patients, also allowing significant cost saving for the health care system.

\section{References}

[1] Reddy, S., Niewiadomska-Bugaj, M., Reddy, Y.V., Galfalvy, H.C., Jagannathan, V., Raman, R., Srinivas, K., Shank, R., Davis, T., Friedman, S., Merkin, B. \& Kilkenny, M., Experiences with ARTEMIS-an Internetbased telemedicine system. Proc AMIA Annu Fall Symp, pp. 759-763, 1997.

[2] Hersh, D., Hersch, F., Mikuletic, L. \& Neilson, S., A Web-based approach to low-cost telemedicine. J Telemed Telecare, 9 Suppl 2, pp. S24-S26, 2003. 
[3] Barbosa, A.K., de A Novaes, M. \& de Vasconcelos, A.M., A web application to support telemedicine services in Brazil. AMIA Annu Symp Proc, pp. 56-60, 2003.

[4] Grasso, V., What works. Web-enabled Telemedicine project developed to monitor the health of Mt. Everest climbers. Health Manag Technol, 20(2), pp. 18, 1999.

[5] Bangert, D., Doktor, R. \& Johnson, E., Designing Web-based telemedicine training for military health care providers. $J$ Contin Educ Health Prof, 21(3), pp. 162-169, 2001.

[6] Finkelstein, J., Hripcsak, G. \& Cabrera, M.R., Patients' acceptance of Internet-based home asthma telemonitoring. Proc AMIA Symp, pp. 336-340, 1998.

[7] Gray, J.E., Safran, C., Davis, R.B., Pompilio-Weitzner, G., Stewart, J.E., Zaccagnini, L. \& Pursley, D., Baby CareLink: using the internet and telemedicine to improve care for high-risk infants. Pediatrics, 106(6), pp. 1318-1324, 2000.

[8] Bellazzi, R., Montani, S., Riva, A. \& Stefanelli, M., Web-based telemedicine systems for home-care: technical issues and experiences. Comput Methods Programs Biomed, 64(3), pp. 175-187, 2001.

[9] Lau, C., Churchill, R.S., Kim, J., Matsen, F.A. $3^{\text {rd }}$ \& Kim, Y., Asynchronous web-based patient-centered home telemedicine system. IEEE Trans Biomed Eng, 49(12), pp. 1452-62, 2002.

[10] Chan, D.S., Callahan, C.W., Sheets, S.J., Moreno, C.N. \& Malone, F.J., An Internet-based store-and-forward video home telehealth system for improving asthma outcomes in children. Am J Health Syst Pharm, 60(19), pp. 1976-1981, 2003.

[11] Mair, F.S., Wilkinson, M., Bonnar, S.A., Wootton, R. \& Angus, R.M., The role of telecare in the management of exacerbations of chronic obstructive pulmonary disease in the home. J Telemed Telecare, 5 Suppl 1, pp. S66-S67, 1999.

[12] Swartz, D., Insuring the security of Internet-based telemedicine systems. Telemed Today, 6(1), pp. 27-29, 1998.

[13] Gutierrez, G., Medicare, the Internet, and the future of telemedicine. Crit Care Med, 29(8 Suppl), pp. N144-N150, 2001.

[14] Tura, A., Quareni, L., Longo, D. \& Badanai M., Improved home care activities for brain-injured children: the creation of a wireless platform. Simulations in Biomedicine V, eds. Z.M. Arnez, C.A. Brebbia, F. Solina, V. Stankovski, WIT Press: Southampton and Boston, pp. 487-495, 2003.

[15] Tura, A., Badanai, M., Longo, D. \& Quareni L., A multi-functional, portable device with wireless transmission for home monitoring of children with a learning disability. $J$ Telemed Telecare, 10(5), pp. 298-302, 2004. 